\title{
Conic projections of the triaxial ellipsoid: the projections for regional mapping of celestial bodies
}

\author{
Maxim V. Nyrtsov, ${ }^{a}$ Maria E. Fleis, ${ }^{b}$ Michael M. Borisov, ${ }^{b}$ Philip J. Stooke ${ }^{c}$ \\ ${ }^{a}$ Lomonosov Moscow State University, Moscow, Russia; nyrtsovmaxim@gmail.com \\ ${ }^{b}$ Institute of Geography, Russian Academy of Sciences, Moscow, Russia; fleis.maria@yandex.ru \\ ${ }^{c}$ The University of Western Ontario, London, ON, Canada; pjstooke@uwo.ca
}

\begin{abstract}
Different distortion classes of the azimuthal and cylindrical projections of the triaxial ellipsoid have been considered in our previous works. These projections make it possible to construct maps of the celestial bodies in planetary scale. However, for regions in the middle latitudes it is advisable to use a conic projection which was not developed until now. In this investigation we describe the development of three conic projections of a triaxial ellipsoid: a conic projection with true scale along meridians, an equal-area conic projection and a quasi-conformal conic projection. In derivation of the projections we use an elliptical cone tangent to a triaxial ellipsoid. The projections are calculated and maps in these projections are created for the first time.
\end{abstract}

Keywords: coordinate system, triaxial ellipsoid, map projection, conic projection, Gaussian coefficients

\section{Introduction}

Nowadays we have examples of regional mapping of celestial bodies in conic projections. For instance the Atlas of the asteroid Vesta available on the Internet (Vesta Atlas Gallery. Jet Propulsion Laboratory). Maps in this atlas were compiled in the Lambert conformal conic projection for the sphere despite the fact that the shape of asteroid is approximated by a triaxial ellipsoid. If the compression of shape of a celestial body is more than $40 \%$ the difference in the conic projections of sphere and ellipsoid will be significant and the reasonability of using of a triaxial ellipsoid is justified. The asteroid (951) Gaspra, the asteroid (243) Ida and the asteroid (433) Eros are examples of such bodies.

For triaxial ellipsoid we assume that conic projections are projections with the meridians as a set of straight lines drawn from a single point, and parallels are curves constructed in accordance with the selected character of distortion. Herewith angles at the crossing points of meridians on the projection depend functionally on corresponding angles on the ellipsoid and of the cone parameters. This definition of conic projections allows us to connect the different classes of projections of a triaxial ellipsoid in a united system.

In this study we present the development of three conic projections of a triaxial ellipsoid with use of an elliptical tan- gent cone:

- conic projection with true scale along meridians;

- equal-area conic projection;

- quasi-conformal conic projection close to the conformal projection in the neighborhood of each meridian corresponding to meridian section which we call the projection of the meridian section.

\section{Derivation of conic projections}

For the derivation of conic projections we use a threedimensional rectangular coordinate system $x, y, z$ with the

origin at the center of the triaxial ellipsoid and associated system of planetocentric angular coordinates $\Phi, \lambda$ on the ellipsoid surface. Let us mention that planetocentric latitude is the angle between the vector $\boldsymbol{r}$ coming from the center of the ellipsoid and the equatorial plane and the longitude is the angle between the plane of the meridian section and the plane of the prime meridian The surface of a triaxial ellipsoid is described by an equation that determines the length of the vector $\boldsymbol{r}$ as a function of latitude and longitude for given values of the semi-axes a, $\mathrm{b}, \mathrm{c}$ and calculated thereon eccentricity of the ellipse of the equatorial section e1 and eccentricity of the ellipse of the prime meridian section $e$.

To derive formulas for conic projections we need elements of the infinitesimal trapezium on the ellipsoid corresponding to given increments of latitude $d \Phi$ and longitude $d \lambda$ in order to compare them with the same elements on the projection plane (see Fig. 1,2). With an accuracy of up to infinitesimal values of a higher-order, the trapezium can be accepted as an infinitesimal parallelogram.

Sides of the parallelogram are differential of the arc of the meridian and the differential of the arc of parallel. Let $\omega$ be the angle between them. All these parameters are expressed by Gaussian coefficients, which we obtained 
using the increment of the vector $r$ along the curve on the surface of the ellipsoid (Nyrtsov et al., 2015).

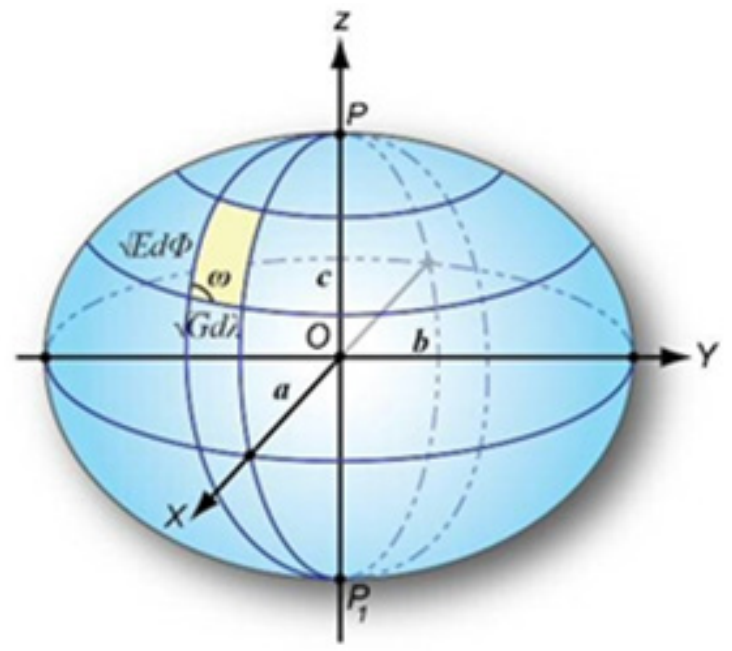

Fig. 1. An infinitesimal trapezium on the surface of ellipsoid.

In the projection plane we use a polar coordinate system $\rho, \delta$ with the pole at the crossing point of the meridians and polar axis which corresponds to the prime meridian and the positive direction counterclockwise. Also we use a rectangular system which $X_{\text {proj }}$ coordinate axis is directed horizontally to the right and $Y_{\text {proj }}$ coordinate axis is directed vertically upward along the central meridian of a map with the origin at the intersection of the meridian and chosen parallel.

Planar rectangular coordinates in projection are:

$$
x_{p r o j}=\rho \sin \delta, \quad y_{\text {proj }}=\rho_{\text {origin }}-\rho \cos \delta,
$$

where the polar distance along the central meridian

$$
\rho_{\text {origin }}=\rho\left(\Phi_{\text {origin }}, \lambda_{0}\right)
$$

is equal to distance between an origin of polar coordinate system and the origin of planar rectangular coordinate system.

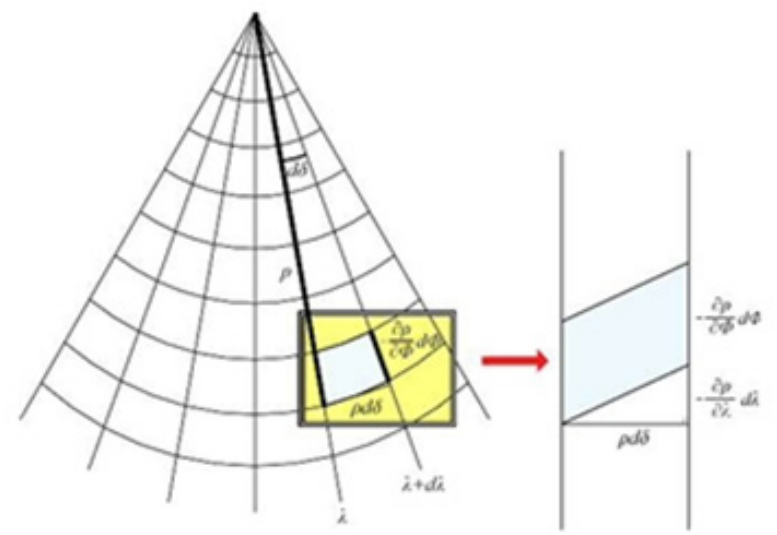

Fig. 2. Infinitesimal trapezoid (parallelogram) in conic projection.

For the derivation of conic projections we obtain the tangent cone parameters and relation of the angle $\delta$ at the vertex of the cone and longitude on the ellipsoid. The formula for calculation of the angle $\delta$ :

$$
\delta=\int_{\lambda_{0}}^{\lambda_{i}} \alpha d \lambda
$$

, where $\alpha$ is the function of longitude and the cone parameter.

We derive formulas of the polar distance for all three offered projections. For conic projections with true scale along meridians

$$
\rho_{i}=\int_{\phi_{i}}^{\varphi_{i}} E d \Phi+L
$$

For equal-area conic projection

$$
\rho_{i}=L+2 \int_{0}^{\phi_{*}} E G \sin \omega d \Phi
$$

For the conic projection of the meridian section:

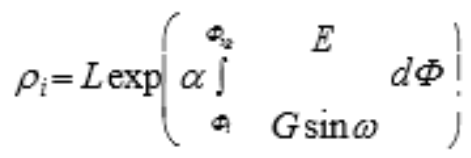

Here $L$ is the length of the tangent line segment from the tangent point to the vertex of the cone; $\Phi_{t g}$ - the latitude of the tangent point

After all we calculate distortions in conic projections. Ellipses of distortion (Tissot indicatrix) for conic projections are shown in Figure 3. Meridians and a line of a tangency of a cone and triaxial ellipsoid do not depend on character of distortions unlike to parallels. Ellipses are connected to the cartographic grid with the parameters of the asteroid 433 Eros and with the direction of counting of longitude goes to the west.
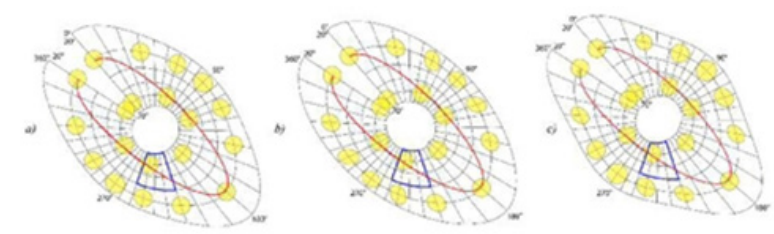

Fig. 3. Tissot indicatrix for a) conic projection with true scale along meridians, b) equal-area conic projection, c) conic projection of the meridian section. Red color line is a line of tangency of a cone. Blue color trapeze is an area of map depicted in the Fig. 4

\section{Mapping of asteroid 433 Eros in conic projections}

The asteroid 433 Eros was taken for approbation of conic projections of the triaxial ellipsoid. It is approximated by a triaxial ellipsoid with semi-axes $\mathrm{a}=17 \mathrm{~km}, \mathrm{~b}=5.5 \mathrm{~km}$, $\mathrm{c}=5.5 \mathrm{~km}$ (Report of the IAU Working Group, 2009). Maps were created in conic projection with true scale along meridians, equal-area conic projection, conic projection of the meridian section for region with craters Casanova and Pelléas from $40^{\circ}$ to $70^{\circ}$ latitude and from $210^{\circ}$ to $250^{\circ}$ longitude. Central meridian of map is $229^{\circ}$,

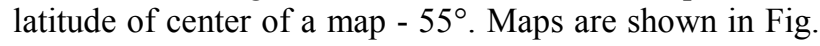
7. To show the asteroid's sur- face we used photo mosaic 
prepared by Philip J. Stooke and available through the Small Bodies Node of NASA's Planetary Data System.
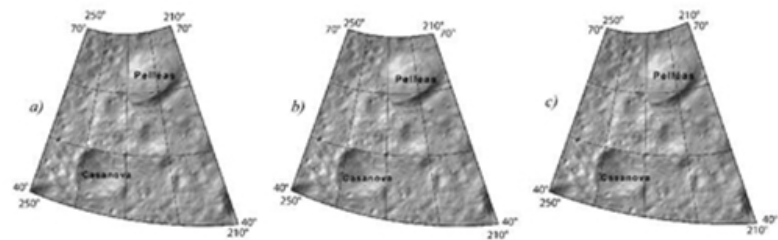

Fig. 4. Maps of asteroid 433 Eros in a) conic projection with true scale along meridians, b) equal-area conic projection, c) conic projection of the meridian section.

\section{Conclusion}

In the article we described derivation of formulas for three conic projections of triaxial ellipsoid for mapping of the territories in the middle latitudes of celestial body. For the triaxial ellipsoid as a surface which can be projected on a plane without distortions we use a direct elliptic cone tangent to ellipsoid.

The polar coordinates system is used for derivation of projections. The polar angle is obtained by integration on a longitude of an increment of this angle depending on an increment of a longitude. The polar distance for all three offered projections is obtained by integration on a meridian that is with the account of an increment of latitude at the fixed value of a longitude. The calculation of the coordinates of the nodes of the cartographic graticule and values of distortions of offered projections are realized in the GIS Research Centre Site by IG RAS (GIS Research Centre Site, Conic projections, 2017).

\section{References}

B. A. Archinal et al Report of the IAU Working Group on Cartographic Coordinates and Rotational Elements// Celest Mech Dyn Astr. Springer, 2010

GIS Research Centre of the Institute of Geography of the Russian Academy of Sciences. Cartographical Projections of Triaxial Ellipsoid. Conic projections. http://geocnt.geonet.ru/en/3_axial_conic Accessed 20 February 2017

Maxim V. Nyrtsov, Maria E. Fleis, Michael M. Borisov and Philip J. Stooke. Equal-Area Projections of the Triaxial Ellipsoid: First Time Derivation and Implementation of Cylindrical and Azimuthal Projections for Small Solar System Bodies// The Cartographic Journal. In- ternational Cartographic Conference, Rio 2015 - Special Issue, May 2015, Vol. 52, No.2, pp.114-124

Vesta Atlas Gallery. Jet propulsion Labaratory. California Institute Of Technology. http://dawn.jpl.nasa.gov/multimedia/vesta atlas_gallery.asp Accessed 20 February 2017 